\title{
Beneficios de la podometría en la prevención de factores de riesgo cardiovascular
}

\author{
DOI 10.5377/alerta.v4i3.10315
}

Carolina Elizabeth Ortíz Laínez ${ }^{1^{*}}$, Karla Rosseline Reyes Mejía ${ }^{2}$, William Andrés Hoyos Arango ${ }^{3}$

1, 2 y 3. Facultad Ciencias de la Salud “Dr. Luis Edmundo Vásquez”, Universidad Dr. José Matías Delgado, La Libertad, El Salvador 3. Ministerio de Salud de El Salvador, San Salvador, El Salvador

${ }^{*}$ Correspondencia

$\nabla$ caro.tiz18@gmail.com

(1) 0000-0002-1278-1508

\begin{abstract}
Resumen
Las enfermedades cardiovasculares constituyen la primera causa de mortalidad mundial debido al efecto sinérgico entre sus factores de riesgo modificables. La actividad física, específicamente la caminata diaria, en conjunto con el podómetro, está asociada a la reducción significativa de eventos cardiovasculares y mortalidad prematura. Se pretende describir los beneficios de la caminata cuantificada por podometría en la prevención de factores de riesgo cardiovascular modificables. Se realizó una revisión a partir de la lectura de artículos originales, de revisión y meta-análisis, utilizando las bases de datos PubMed e HINARI y el buscador Google Académico. se utilizó la ecuación de búsqueda «podometría AND hipertensión» con obsolescencia menor de 5 años en el 86 \% de referencias. Se demuestra que caminar con podómetro promueve un incremento de actividad física diaria al brindar motivación mediante retroalimentación positiva inmediata. Los beneficios cardiovasculares pueden obtenerse realizando al menos 10000 pasos diarios y consisten en la mejora de la presión arterial, glicemia, perfil lipídico y la composición corporal. La caminata diaria con podómetro promueve hábitos saludables y previene la aparición o progresión de factores de riesgo cardiovascular modificables, principalmente en personas con morbilidad previa.

Palabras clave

Caminata, riesgo cardiovascular, inactividad física, hipertensión, diabetes mellitus tipo 2.
\end{abstract}

\section{Abstract}

Cardiovascular diseases constitute the first cause of mortality worldwide because of the synergic effect between their modifiable risk factors. Physical activity, specifically daily walking with the use of a pedometer, is associated with a meaningful reduction of cardiovascular events and premature mortality. The purpose of this review is to describe the benefits of walking-based-pedometer in the prevention of modifiable cardiovascular risk factors. Original articles, review articles, and meta-analysis, were reviewed using PubMed and HINARI data bases, as well as Google Scholar, introducing the following search equation «pedometry AND hypertension». An obsolescence minus 5 years in the $86 \%$ of references was used. It is shown that walking-based-pedometer promotes an uprising in daily physical activity by promoting selfmotivation mediated by immediate positive feedback; besides, it encourages healthier behavior. Cardiovascular benefits can be obtained by realizing at least 10000 steps daily and consist on the improvement of blood pressure, glycaemia, lipid panel and body composition. Daily walking-based-pedometer promotes healthier habits and thus prevents the emergence and progression of modifiable risk factors, mainly on people with previous morbidity.

Keywords

Walking, cardiovascular risk, physical inactivity, hypertension, type 2 diabetes.

\section{Introducción}

Las enfermedades cardiovasculares (ECV) constituyen la primera causa de mortalidad a nivel mundial', emergiendo como uno de los principales problemas de salud pública para los países en vías de desarrollo² debido a su aparición temprana y al efecto sinérgico entre sus factores de riesgo modificables. En consecuencia, es importante promover el cese a dicha exposición ${ }^{3}$. f ACCESO ABIERTO

Benefits of pedometry in the prevention of cardiovascular risk factors

Citación recomendada: Ortíz Laínez CE, Reyes Mejía $\mathrm{KR}$, Hoyos Arango WA. Beneficios de la podometría en la prevención de factores de riesgo cardiovascular. Alerta. 2021;4(3):151-158. DOI 10.5377/alerta.v4i3.10315

Recibido:

30 de octubre de 2020

\section{Aceptado:}

19 de julio de 2021

\section{Publicado:}

26 de julio de 2021

\section{Contribución de autoría:} OLCE'1, RMKR²: Concepción, metodología, adquisición, análisis e interpretación de los datos OLCE', RMKR², HAWA3: Diseño del estudio y la revisión crítica del contenido intelectual.

\section{Conflicto de intereses:}

Los autores declaran no tener conflicto de intereses. 
El riesgo cardiovascular depende del número de factores presentes en cada individuo ${ }^{2}$. Entre los modificables se encuentran hábitos no saludables como el tabaquismo, abuso de alcohol, mala alimentación, conducta sedentaria e inactividad física ${ }^{4}$. Al ser modificados tiene un impacto sobre la hipertensión arterial, la dislipidemia, el sobrepeso, obesidad y anormalidades en el metabolismo de la glucosa 5 .

Según la Organización Mundial de la Salud (OMS), la inactividad física constituye uno de los factores de riesgo que más contribuye a la mortalidad global, siendo aproximadamente 3,2 millones de muertes debido a enfermedades cardiovasculares, diabetes, hipertensión, entre otros ${ }^{6}$. Mientras que la realización de actividad física (AF) es una medida eficaz de prevención primaria y secundaria en más de 25 enfermedades crónicas, entre ellas las ECV ${ }^{7}$. Sin embargo, aunque se conocen los beneficios que brinda a la salud, la mayoría de personas presentan dificultades para involucrarse en ejercicios estructurados y supervisados comparado con lo que se realiza dentro de la rutina diaria8.

Por consiguiente, la caminata, al ser considerada como la forma más natural de realizar actividad física, es la opción ideal al poder incorporarse fácilmente a diario y proveer dinamismo a la actividad aeróbica en personas sedentarias ${ }^{9,10}$. En conjunto con herramientas, como el podómetro, está asociada a la reducción significativa del riesgo de mortalidad prematura y prevenir enfermedades crónicas ${ }^{11}$.

Al ser un instrumento práctico y accesible para la población en general, el podómetro cuantifica el número de pasos y es usado para realizar programas de intervención basados en caminata ${ }^{12}$, ya que resulta efectivo al brindar motivación e incrementar la actividad física en personas con alguna morbilidad crónica y así beneficiar su salud ${ }^{13}$.

Se realizó una revisión a partir de la lectura de artículos originales, de revisión, metaanálisis, estudios retrospectivos, prospectivos, casos y controles en los idiomas inglés y español. Se consultó la Biblioteca Nacional de Medicina de los Estados Unidos mediante la base de datos electrónica PubMed, HINARI y el buscador Google Académico, para la obtención de información científica. Se limitó la búsqueda de artículos con una obsolescencia menor de 5 años en el 86\% de las referencias. Se utilizaron términos de búsqueda más el operador booleano «AND» como «podometría AND hipertensión», «podómetro AND caminata», «podometría AND diabetes mellitus», «podometría AND alimentación saludable» y «podometría AND tabaco».
Por lo tanto, el propósito de esta revisión es describir los beneficios de la caminata cuantificada por podometría en la prevención de factores de riesgo cardiovascular modificables.

\section{Desarrollo}

\section{Uso de podómetros durante la caminata y la adquisición de hábi- tos saludables}

Según Liu et al., la adherencia a estilos de vida saludable como tener una dieta adecuada, no fumar, beber alcohol con moderación y realizar AF de intensidad moderada ( $\geq 150 \mathrm{~min} /$ semana), se relaciona con una reducción del $14 \%$ del riesgo cardiovascular y una reducción del $27 \%$ de la mortalidad por ECV ${ }^{14}$.

En este sentido, la evidencia es concluyente en cuanto a los beneficios de realizar AF de forma regular, por ello ha sido importante emplear métodos objetivos para poder medirla mediante podómetros y acelerómetros ${ }^{15}$. Los podómetros son dispositivos con sensores de movimiento que tienen la finalidad de registrar los pasos diarios al caminar o correr. Son instrumentos pequeños, ligeros, no intrusivos, fáciles de utilizar y los pasos acumulados pueden visualizarse digitalmente en una pantalla ${ }^{16}$.

Los primeros podómetros, diseñados para llevarlo en la cintura, incorporan un brazo de palanca horizontal suspendido por un resorte que se mueve hacia arriba y hacia abajo con cada paso. Dicho movimiento abre y cierra un circuito eléctrico, lo que hace que el dispositivo registre los pasos ${ }^{17}$. Los podómetros con un cristal piezoeléctrico utilizan la fuerza mecánica del movimiento corporal para generar una carga eléctrica para contar los pasos. En general, han surgido una amplia variedad de contadores de pasos incluidos los acelerómetros triaxiales e interfaces de teléfonos inteligentes ${ }^{18}$.

En cuanto a su precisión y fiabilidad, estos pueden variar en función del mecanismo interno, la velocidad de los pasos y su ubicación en el cuerpo (si se llevan en la cadera, muñeca, tobillo e incluso en el bolsillo). Los contadores de pasos basados en acelerómetros que se usan en la cintura son generalmente más precisos que los podómetros con palanca de resorte. Además, estos últimos disminuyen su precisión en personas obesas. El conteo insuficiente a bajas velocidades es un problema que suele afectar a algunos contadores de pasos. Por lo que en países como Japón han establecido normas industriales (tener una precisión de $\pm 3 \%$ para su aprobación) ${ }^{17}$. 
En consecuencia, estos dispositivos se han convertido en herramientas útiles no solo para el registro sino también para la motivación de los comportamientos relacionados con la AF. Esto se debe a que proporcionan una retroalimentación inmediata al usuario, al visualizar el número de pasos dados en el día, permitiendo el cumplimiento de objetivos individuales para estimularlos a alcanzar un mayor número de pasos ${ }^{19}$. Eso demuestra que pueden generar beneficios en la composición corporal y otros parámetros que contribuyen a la salud cardiovascular ${ }^{13}$.

Fundamentándose en que las principales razones porque no se realiza AF son la falta de tiempo y la motivación, Miragall et al. en su ensayo aleatorizado con 75 adultos jóvenes sedentarios, realizaron una intervención motivacional basada en internet (IMI) apoyada con podómetros y mostraron que los pasos diarios promedio en el grupo de intervención fueron significativamente más altos que en el grupo control, incrementando un promedio de 2000 - 2200 pasos por día. Se concluyó que los podómetros fueron el componente que mayormente estimuló los cambios en el comportamiento en los jóvenes sedentarios ${ }^{20}$.

Por otra parte, este tipo de intervenciones ayuda a alcanzar las recomendaciones mínimas sobre la actividad física primaria en adultos que, según las Physical Activity Guidelines for Americans, consiste en realizar ejercicio aeróbico de moderada intensidad de 150-300 minutos por semana ${ }^{21}, 30$ minutos por 5 días a la semana o 50 minutos por 3 días a la semana ${ }^{22}$

Tal como en el estudio de cohorte prospectivo de Jilcott et al. en adultos mayores de áreas rurales del Sureste de los Estados Unidos, se realizó una intervención sobre alimentación saludable y el aumento de la actividad física usando podómetros en 249 participantes. Se observó que con un promedio de 4525 pasos al día lograron 160 minutos de actividad física moderada a la semana, durante la intervención de 6 meses $^{23}$.

En este sentido, Tudor Locke et al., en un estudio prospectivo, establecieron la conversión entre el número de pasos diarios con el tiempo empleado en actividad física diaria de intensidad moderada a vigorosa (AFMV). Incluyeron a 3523 adultos jóvenes a quienes se les proporcionó un acelerómetro ActiGraph para recopilar el número de pasos por al menos un día válido (al menos 10 h en un día) durante 7 días y concluyeron que acumular 8000 pasos al día era un buen indicador de 30 min AFMV y 7000 pasos todos los días de la semana equivalía a 150 minutos a la semana ${ }^{24}$.
Cabe mencionar que desde que inició el auge del uso de podómetros para la cuantificación de pasos en 1965, se recomendaba el objetivo de caminar al menos 10000 pasos al día para obtener beneficios a la salud de sus usuarios. Sin embargo, en los últimos años la evidencia sobre esta afirmación no ha sido consistente ${ }^{19}$. Además, en los estudios revisados los participantes se han visto beneficiados al menos con 10000 pasos diarios; inclusive se ha obtenido una disminución en las tasas de mortalidad.

Tal como en el estudio de cohorte prospectivo de Lee et al., que incluyó a 16741 mujeres adultas mayores estadounidenses, se evaluó las asociaciones del número de pasos/día y su intensidad en relación con la mortalidad por todas las causas. Los participantes usaron los acelerómetros ActiGraph durante al menos 10 horas diarias por semana durante los años de seguimiento. Se observó que aproximadamente 4400 pasos/ día se asociaban a una reducción del 41\% en la tasa de mortalidad en comparación con aproximadamente 2700 pasos/día y, a su vez, hubo una disminución constante en la misma con más pasos acumulados hasta los 7500 pasos/día, más allá de los cuales ya no se manifestaron cambios ${ }^{25}$.

También, en el ensayo aleatorizado de Fayehun et al. se dividieron a 46 participantes nigerianos con diabetes mellitus tipo 2 (DM2) en un grupo de intervención (Gl), estableciéndoles la meta de realizar 10000 pasos diarios durante 10 semanas. Sin embargo, sólo el 6,1\% de los participantes del Gl logró el objetivo (promedio fue 6507 pasos/día) y a pesar de la baja adherencia a la prescripción hubo mejora en el control glicémico de los participantes ${ }^{26}$.

Las intervenciones en las que se combina la dieta y AF han evidenciado proveer más beneficios a la salud cardiovascular y en reducir el riesgo de mortalidad, que con cualquiera de las intervenciones por sí solas $^{27}$. Por lo que algunos investigadores en sus intervenciones con podómetros han implementado dietas saludables, obteniendo resultados favorables.

Yu et al., en un ensayo clínico, evaluaron la efectividad de la caminata con podómetro sobre el manejo del peso y otros parámetros en salud en una población china ocupacional. Incluyeron a 688 participantes y realizaron tres sesiones de caminata de 30 a 60 minutos por 3 meses. En los participantes con sobrepeso u obesidad se obtuvo una disminución en el Índice de Masa Corporal (IMC), circunferencia de cintura (CC) y porcentaje de grasa corporal (\%GC) del $2,2 \%, 1,8 \%$ y $3,3 \%$, respectivamente. Pero no hubo cambios significativos en el HDL ni tri- 
glicéridos. Los autores sugirieron que pudo deberse a la falta de un régimen dietético estricto durante la intervención ${ }^{28}$.

Oftedal et al., en su estudio de cohorte prospectivo que incluyó 1697 participantes adultos mayores australianos para evaluar la asociación entre el recuento de pasos y la calidad de la dieta en relación con la mortalidad por todas las causas, demostraron que un incremento de 1000 pasos diarios se asocia a una reducción del 7\% del riesgo de mortalidad y una mejor calidad de la dieta se relaciona con una reducción del 14\% en el riesgo de mortalidad, en un seguimiento de 9,6 años ${ }^{29}$.

Al contar con la compresión de los participantes sobre el uso de dispositivos móviles con función de podómetro ha demostrado resultados significativos al mejorar la actividad física diaria y promover dietas más saludables, tal como lo demuestra Walsh et al. con la aplicación Accupedo-Pro Pedometer en una población adulta joven ${ }^{30}$.

Alonso et al., en su ensayo clínico aleatorizado, buscaron aumentar la AF y mejorar los hábitos alimenticios en personas con DM2. Ejecutaron una intervención con la aplicación (EVIDENT II) ${ }^{31}$ con función de podómetro y emplearon menús nutritivos sobre dieta mediterránea a los 204 participantes. El Gl aumentó 1852 pasos al día y también disminuyeron su tiempo sedentario en 34,3 minutos por día a la semana a los 3 meses de seguimiento; a los 12 meses, el número de pasos persistió con aumentos significativos en general ${ }^{32}$.

En cuanto al hábito tabáquico, hay evidencia que sesiones cortas de actividad física reducen el fumado y el estado de ánimo negativo causado por la abstinencia a la nicotina ${ }^{33}$.

En el estudio controlado aleatorizado de Nair et al. participaron 101 fumadores de escasos recursos con inactividad física. Al Gl Step Up To Quit SUTQ se le brindó consejería semanal vía telefónica e incentivó a realizar 7500 pasos diarios, con la finalidad de utilizar la AF diaria como método primario para manejar la urgencia de fumar y al GC se le implementó la consejería convencional. Se les proporcionó un podómetro y la semana previa al quit day el $80 \%$ de los integrantes del grupo SUTQ había alcanzado la meta, comparado con un 20\% del grupo controp3.

Los efectos se mantuvieron una semana post-quit day (81 vs 19\%) y al mes de seguimiento (80 vs 20\%). Además, el 32,4\% de participantes del grupo SUTQ reportó dejar el tabaco. Se concluyó que una intervención integral constituida por consejería y caminata diaria tiene el potencial de promover hábitos saludables en los tabaquistas ${ }^{33}$.

\section{Impacto sobre los factores de riesgo cardiovascular}

El ejercicio aeróbico, en este caso la caminata, es recomendado como método antihipertensivo de primera línea ${ }^{34,}$ debido a que la presión arterial (PA) es más baja en los días en que se ejecuta que en aquellos en los que no se efectúa ${ }^{35}$. Esta respuesta fisiológica, descrita por primera vez por Fitzgerald en 198136, se conoce como hipotensión post-ejercicio (HPE). Dicho término consiste en la disminución inmediata de la PA posterior a la realización de una sesión de actividad física aeróbica, la cual presenta una duración de 24 horas posterior a su ejecución ${ }^{37}$. Además, se sugiere que la sumatoria de sesiones individuales de ejercicio aeróbico con HPE subsecuente puede conllevar a la disminución a largo plazo de la presión arterial ${ }^{38}$, por lo que se infiere que la HPE puede ser un predictor de los efectos de dicha AF sobre la $\mathrm{PA}^{39}$.

En el artículo original de Kobayashi et al., se reclutaron 44 hombres japoneses sedentarios y se les entregó un podómetro, instruyéndolos a caminar y recopilar el número de pasos diarios por 50 días consecutivos, sin intervención dietética. El promedio de pasos diario realizados al finalizar el estudio fue de 8211 . Se obtuvo una disminución de $10 \mathrm{mmHg}$ (124 vs $114 \mathrm{mmHg}$ ) en la presión arterial sistólica (PAS) al haber hecho $\geq 8000$ pasos diarios40. Mientras que en el estudio de $\mathrm{Yu}$ et al., tras la caminata personalizada por 3 meses, los participantes con sobrepeso u obesidad manifestaron una disminución de la PAS y presión arterial diastólica (PAD) de $3,3 \mathrm{mmHg}$ y $3,2 \mathrm{mmHg}$, respectivamente ${ }^{28}$.

Está establecido que los valores de la PAS y PAD están inversamente correlacionados con el número de pasos diarios cuantificados con podómetro ${ }^{19}$, al reducir la resistencia vascular periférica sin el incremento del gasto $\mathrm{cardíaco}^{37}$, ya que caminar regularmente promueve la función endotelial al aumentar la biodisponibilidad y síntesis del óxido nítrico, disminuye el tono vasoconstrictor mediado por la endotelina-1 y la elevada actividad del sistema nervioso simpático ${ }^{41}$.

Por otro lado, el estudio cuasi experimental pre-post intervención de Hasan et al. examinó los efectos de caminata con podómetro en 52 mujeres jóvenes, dividiéndolas en dos grupos según su IMC normal o elevado y se les brindó un podómetro, instándoles a caminar diariamente por 9 semanas. La media de pasos realizados en general fue de 7056 diarios. En el caso de la PAS y PAD, en ambos grupos, no se evidenciaron cambios significativos (113,79 vs $114,4 \mathrm{mmHg}$ ) 
y $(75,38$ vs $74,9 \mathrm{mmHg})$, respectivamente, debido a que todas las participantes eran normotensas $^{8}$. Esto se explica mediante la ley de valores iniciales donde a mayor presión arterial en reposo mayor disminución se presenta tras la actividad física ${ }^{35}$.

La caminata, como una actividad aeróbica de leve a moderada intensidad ${ }^{35}$, constituye una ventaja para las personas con sobrepeso u obesidad porque se encuentran asociadas a una baja intensidad de esfuerzo y, por lo tanto, presentan valores más bajos de gasto energético, intensidad metabólica y cantidad de ejercicio al querer iniciar actividad física con ejercicios que requieren intensidad moderada a vigorosa ${ }^{42}$.

Por eso se recomienda esta actividad monitoreada con podómetro porque al observar la cuantificación de pasos y recibir una retroalimentación inmediata, incentiva a realizar AF diaria y aumentar gradualmente el número de pasos diarios, provocando así un gasto energético constante ${ }^{8}$. A diferencia de la población con IMC normal, ya que presentan una mejor condición física, requieren de una actividad de mayor intensidad con el fin de obtener resultados significativos ${ }^{43}$.

Tal es el caso en el estudio de Hasan et al., donde se observó que los voluntarios con sobrepeso u obesidad manifestaron una alteración significativa en los niveles de triglicéridos (TG) disminuyendo 9,1 mg/dl, comparado con el grupo con IMC normal con disminución de 3,42 mg/dl, posterior a la intervención. Además, quienes realizaron > 7065 pasos diarios, siendo la mayoría con un IMC normal, manifestaron solo un cambio significativo dentro de los parámetros evaluados ${ }^{8}$.

Una de las limitantes de Kobayashi et al. fue la ausencia de cambios significativos en el perfil lipídico de sus participantes, exceptuando los TG (119 vs 101 mg/dl), la cual puede ser explicada por la reducción leve y casi nula del IMC, \%GC y la CC, a diferencia de como se observa en Hasan et al. que presentaron cambios significativos en estos últimos parámetros $(p<0,01)$, reduciendo los niveles de lipoproteína de baja densidad y aumentando la adiponectina de alto peso molecular en 4,63 mg/dl y 0,07 $\mathrm{\mu g} / \mathrm{ml}$, respectivamente ${ }^{8,40}$.

La reducción de la obesidad central, especialmente la CC, provoca el aumento en la producción de la adiponectina, una adipocitocina principalmente secretada por los adipocitos, con la cual manifiesta una correlación inversa. Esta última posee un efecto antiaterogénico, antiinflamatorio y promueve la sensibilización a la insulina ${ }^{8,40,44}$.

En cuanto al control glicémico, se ha encontrado que el uso de podómetros pro- porciona una disminución significativa de la hemoglobina glicosilada (HbA1c), siendo el mejor indicador de los niveles crónicos de glicemia y del riesgo de complicaciones a largo plazo ${ }^{45}$.

Por ejemplo, en el ensayo de Fayehun et al., se les entregó un podómetro electrónico Digi-Walker SW-200 y se usó el análisis de covarianza de ANCOVA para ajustar la HbA 1c basal y comparar su alteración entre ambos grupos posterior a la intervención. La $\mathrm{HbA} 1 \mathrm{c}$ basal era de 6,6\% en general, sin embargo, el Gl presentó una reducción final de 0,74\% comparado con el GC, concluyendo en una reducción significativa en los valores de la HbA1 $c^{26}$.

Yom-Tov et al. realizaron un ensayo de 26 semanas de intervención con 27 adultos sedentarios con DM2 que buscaba incrementar el nivel de AF mediante la función de podómetro en teléfonos inteligentes con retroalimentación individualizada. Se dividieron en un Gl que constó de un plan personalizado y un GC que incluyó recordatorios semanales. Al inicio, el promedio de HbAc1 basal era de 7,8\%, pero esta mejoró un 0,28\% después de la intervención para todos los pacientes ${ }^{46}$.

En el caso del Gl hubo una reducción superior de la HbA1c, indicando que a mayor retroalimentación personalizada por la AF constante, mayor era la reducción en los niveles de glucosa sérica en los participantes del Gl. Se concluyó que un manejo basado en un plan personalizado de caminata diaria puede fomentar la AF en los pacientes con DM2 y mejorar su control glicémico ${ }^{46}$.

Efectos en la resistencia a la insulina han sido evaluados en intervenciones basadas en podómetros mediante el Modelo Homeostático para evaluar la Resistencia a la Insulina (HOMA-IR), la cual se estima con las concentraciones de glucosa e insulina en ayunas y funciona como predictor de riesgo de padecer $\mathrm{DM}^{47}$.

Dasgupta et al. ejecutaron un ensayo controlado aleatorizado con 275 participantes con DM2 o HTA, con los objetivos de promover la AF mediante caminata diaria cuantificada con podómetro y mejorar el control glicémico. Se dividió a los participantes en dos grupos. Al Gl se incentivó llegar a los 3000 pasos diarios en el transcurso de 1 año y al GC se solicitó realizar AF con duración de 30-60 min al día por el mismo periodo ${ }^{48}$.

Los participantes con DM2 dentro del Gl presentaron una disminución de la $\mathrm{HbA} 1 \mathrm{c}$ comparada con los del GC (-0,38\%, 95\% Cl $-0,69$ a -0,06) y una reducción en la resistencia a la insulina en el Gl comparado con el GC (HOMA-IR -0,96, 95\% Cl -1,72 a -0,21). Al final se concluyó que, aunque no se llegó a 
lo estipulado, hubo mejoras en la $\mathrm{HbA1c}$ y en la sensibilidad a la insulina ${ }^{48}$.

Se afirma que al aumentar el ejercicio aeróbico diario reduce el riesgo de resistencia a la insulina, al mejorar su sensibilidad y favorecer la absorción de glucosa en los tejidos. Por ello, la intercesión con caminata cuantificada con podómetro es una estrategia efectiva para la prevención de DM2 y síndrome metabólico ${ }^{49}$.

\section{Conclusiones}

Considerando que la disminución de inactividad física puede prevenir y controlar las enfermedades no transmisibles, se recomienda a la caminata cuantificada con podómetro ya que es un método práctico y seguro en la rutina diaria por su efectividad para estimular cambios en el estilo de vida, al incrementar la actividad física diaria mediante la motivación con metas específicas e individualizadas.

La caminata monitoreada con podómetro es el ejercicio aeróbico de elección para iniciar actividad física en personas sedentarias con sobrepeso u obesidad, ya que los efectos positivos se manifiestan con una baja intensidad de esfuerzo físico.

Se evidenció en los estudios realizados que no es necesario llegar a los 10000 pasos diarios para obtener los beneficios de caminata diaria con podómetro; pero la existencia de una mayor actividad física derivada del uso de podómetro tiene un efecto benéfico sobre los factores de riesgo cardiovascular al prevenir su aparición o progresión.

\section{Financiamiento}

No hubo fuentes de financiamiento.

\section{Referencias bibliográficas}

1. Oficina Regional de las Américas de la Organización Mundial de la Salud. La OMS revela las principales causas de muerte y discapacidad en el mundo: 2000-2019. OPS. 2020. Fecha de consulta: 14 de julio de 2021. Disponible en: https://www.paho. org/es/noticias/9-12-2020-oms-revelaprincipales-causas-muerte-discapacidadmundo-2000-2019

2. Querales M, Rojas S, Romero G, Ramírez J. Estudio piloto de los factores clásicos de riesgo cardiovascular en una comunidad rural del municipio San Diego, Venezuela. Avances en Biomedicina. 2016;5(3):138-48. Disponible en: https://www.redalyc.org/ jatsRepo/3313/331349259004/index.html
3. Ferreira D, Díaz M, Bonilla C. Factores de riesgo cardiovascular modificables en adolescentes escolarizados de Ibagué 2013. Rev Fac Nac Salud Pública. 2017;35(2):26473. DOI: 10.17533/udea.rfnsp.v35n2a10

4. Zuni K, More B, Fernández C, García B, Ruiz J, Pérez V. Prevalence of Cardiovascular Risk Factors in Hospitalized Patients in a Lima Hospital. Rev Fac Med Hum. 2019;19(4):6873. DOI: 10.25176/RFMH.v19i4.2343

5. Torres Damas LE. Factores de riesgo aterogénico en población adulta venezolana. Rev Cubana Angiol Cir Vasc. 2016;17(1):44-54. Disponible en: $\underline{\text { http:// }}$ scielo.sld.cu/scielo.php?script=sci_arttext\&p $\mathrm{id}=$ S1682-00372016000100007

6. Sharara E, Akik C, Ghattas H, Makhlouf C. Physical inactivity, gender and culture in Arab countries: a systematic assessment of the literature. BMC Public Health. 2018;18:639-657. DOI: 10.1186/s12889-018$5472-z$

7. Warburton DE, Bredin SS. Reflections on Physical Activity and Health: What Should We Recommend?. Can J Cardiol. 2016;32(4):495-504. DOI: 10.1016/j. cjca.2016.01.024

8. Hasan H, Attlee A, Mohamed HJBJ, Aris N, Muda WAMBW. Counting Footsteps with a Pedometer to Improve HMW Adiponectin and Metabolic Syndrome among Young Female Adults in the United Arab Emirates. Journal of Obesity. 2018;2018:1-9.

DOI: 10.1155/2018/1597840

9. Vetrovsky T, Cupka J, Dudek M, Kuthanova B, Vetrovska K, Capek V, et al. A pedometerbased walking intervention with and without email counseling in general practice: a pilot randomized controlled trial. BMC Public Health. 2018;18(1):635-47. DOI: 10.1186/s12889-018-5520-8

10. Haudhry UAR, Wahlich C, Fortescue R, Cook DG, Knightly R, Harris T. The effects of stepcount monitoring interventions on physical activity: systematic review and metaanalysis of community-based randomised controlled trials in adults. Int J Behav Nutr Phys Act. 2020;17(1):129-145. DOI: 10.1186/ s12966-020-01020-8

11. Hall KS, Hyde ET, Bassett DR, et al. Systematic review of the prospective association of daily step counts with risk of mortality, cardiovascular disease, and dysglycemia. Int J Behav Nutr Phys Act. 2020;17(1):78. DOI: 10.1186/s12966-020-00978-9

12. Baghianimoghaddam MH, Bakhtari-Aghdam F, Asghari-Jafarabadi M, Allahverdipour H, Dabagh-Nikookheslat S, Nourizadeh R. The Effect of a Pedometer-based Program Improvement of Physical Activity in Tabriz University Employees. Int J Prev Med. 2016;1;7:50-55. DOI: 10.4103/20087802.177897 
13. Sheshadri A, Kittiskulnam P, Lai JC, Johansen KL. Effect of a pedometer-based walking intervention on body composition in patients with ESRD: a randomized controlled trial. BMC Nephrol. 2020;21(1):100-108. DOI: 10.1186/s12882-020-01753-5

14. Liu G, Li Y, Hu Y, Zong G, Li S, Rimm E, et al. Influence of Lifestyle on Incident Cardiovascular Disease and Mortality in Patients with Diabetes Mellitus. J Am Coll Cardiol. 2018;71(25):2867-2876. DOI: 10.1016/j.jacc.2018.04.027

15. Benjamin E, Muntner P, Alonso A, Bittencourt M, Callaway C, Carson A, et al. Heart Disease and Stroke Statistics-2019 Update: A Report from the American Heart Association. Circulation. 2019;139(10):e56-e528. DOI: 10.1161/CIR.0000000000000659

16. Miragall M, Domínguez A, Cebolla A, Baños RM. El uso de podómetros para incrementar la actividad física en población adulta: una revisión. Clínica y Salud. 2015;26(2):81-9. DOI: 10.1016/j.clysa.2015.06.002

17. Bassett DR Jr, Toth LP, LaMunion SR, Crouter SE. Step Counting: A Review of Measurement Considerations and Health-Related Applications. Sports Med. 2017;47(7):1303-1315. DOI: 10.1007/s40279016-0663-1

18. Park W, Lee VJ, Ku B, Tanaka H. Effect of walking speed and placement position interactions in determining the accuracy of various newer pedometers. Journal of Exercise Science \& Fitness. 2014;12(1):31-37. DOI: 10.1016/j.jesf.2014.01.003

19. Tudor-Locke C, Bassett D. How Many Steps/ Day Are Enough?. Sports Med. 2004;34(1):18. DOI: 10.2165/00007256-200434010-00001

20. Miragall M, Domínguez A, Navarro J, Cebolla A, Baños R. Increasing physical activity through an Internet-based motivational intervention supported by pedometers in a sample of sedentary students: A randomised controlled trial. Psychology \& Health. 2018;33(4):465-482. DOI: 10.1080/08870446.2017.1368511

21. American College of Sports Medicine. ACSM's guidelines for exercise testing and prescription. 11th Edition. Philadelphia. Chapter 1, Benefits and Risks Associated with Physical Activity. 59-95.

22. American College of Sports Medicine. ACSM's guidelines for exercise testing and prescription. 11th Edition. Philadelphia. Chapter 5, General Principles of Exercise Prescription. 224-55.

23. Pitts SB, Keyserling TC, Johnston LF, Evenson KR, McGuirt JT, Gizlice Z, Whitt OR, Ammerman AS. Examining the association between intervention-related changes in diet, physical activity, and weight as moderated by the food and physical activity environments among rural, Southern Adults.
J Acad Nutr Diet. 2017;117(10):1618-27.

DOI: 10.1016/j.jand.2017.04.012

24. Tudor-Locke C, Leonardi C, Johnson WD, Katzmarzyk PT, Church TS. Accelerometer steps/day translation of moderate-tovigorous activity. Preventive Medicine. 2011;53(1):31-3. DOI: 10.1016/j. ypmed.2011.01.014

25. Lee I, Shiroma E, Kamada M, Bassett D, Matthews C, Buring J. Association of Step Volume and Intensity With All-Cause Mortality in Older Women. JAMA Intern Med. 2019;179(8):1105-1112. DOI: 10.1001/ jamainternmed.2019.0899

26. Fayehun AF, Olowookere $\bigcirc 0$, Ogunbode AM, Adetunji AA, Esan A. Walking prescription of 10000 steps per day in patients with type 2 diabetes mellitus: a randomised trial in Nigerian general practice. Br J Gen Pract. 2018;68(667):e13945. DOI: 10.3399/bjgp18X694613

27. Lanier JB, Bury DC, Richardson SW. Diet and physical activity for cardiovascular disease prevention. Am Fam Physician. 2016;93(11):919-24. Disponible en: https:// www.aafp.org/afp/2016/0601/p919.html

28. Yu Y, Lv Y, Yao B, Duan L, Zhang X, Xie L, et al. A novel prescription pedometerassisted walking intervention and weight management for Chinese occupational population. PLoS One. 2018;13(1):e0190848. DOI: 10.1371/journal.pone.0190848

29. Oftedal S, Holliday EG, Attia J, Brown WJ, Collins CE, Ewald B, Glozier N, McEvoy M, Morgan PJ, Plotnikoff RC, Stamatakis E. Daily steps and diet, but not sleep, are related to mortality in older Australians. Journal of Science and Medicine in Sport. 2020;23(3):276-82. DOl: 10.1016/j. jsams.2019.09.018

30. Walsh JC, Corbett T, Hogan M, Duggan J, McNamara A. An mHealth intervention using a smartphone app to increase walking behavior in young adults: a pilot study. JMIR mHealth and uHealth. 2016;4(3):e109. DOI: 10.2196/mhealth.5227

31. Recio-Rodriguez Jl, Agudo-Conde C, Martin-Cantera C, González-Viejo MN, Fernandez-Alonso MC, Arietaleanizbeaskoa MS, Schmolling-Guinovart Y, MaderueloFernandez JA, Rodriguez-Sanchez E, GomezMarcos MA, Garcia-Ortiz L. Short-term effectiveness of a mobile phone app for increasing physical activity and adherence to the Mediterranean diet in primary care: a randomized controlled trial (EVIDENT II study). J Med Internet Res. 2016;18(12):e331. DOI: 10.2196/jmir.6814

32. Alonso-Domínguez $\mathrm{R}$, Patino-Alonso MC, Sánchez-Aguadero N, García-Ortiz L, Recio-Rodríguez JI, Gómez-Marcos MA. Effect of a multifactorial intervention on the increase in physical activity in 
subjects with type 2 diabetes mellitus: a randomized clinical trial (EMID study). Eur J Cardiovasc Nurs. 2019;18(5):399-409. DOI: 10.1177/1474515119835048

33. Nair US, Patterson F, Rodriguez D, Collins BN. A telephone-based intervention to promote physical activity during smoking cessation: A randomized controlled proof-of-concept study. Translational Behavioral Medicine. 2016; 7(2): 138-47. DOI: 10.1007/s13142-0160449-x

34. Pescatello LS, Schifano ED, Ash GI, Panza GA, Lamberti L, Chen MH, Deshpande V, Zaleski A, Farinatti P, Taylor BA, Thompson PD. Deeptargeted exon sequencing reveals renal polymorphisms associate with postexercise hypotension among African Americans. Physiological Reports. 2016;4(19):e12992. DOI: 10.14814/phy2.12992

35. Pescatello L, MacDonald H, Lamberti L, Johnson B. Exercise for Hypertension: A Prescription Update Integrating Existing Recommendations with Emerging Research. Curr Hypertens Rep. 2015;17(11):87. DOI: 10.1007/s11906-015-0600-y

36. Pescatello LS, MacDonald HV, Lamberti L, Johnson BT. Exercise for hypertension: a prescription update integrating existing recommendations with emerging research. Physiological Reports. 2017; 5(22):e13510. DOI: 10.14814/phy2.13510

37. Cilhoroz BT, Schifano ED, Panza GA, Ash Gl, Corso L, Chen MH, Deshpande V, Zaleski A, Farinatti P, Santos LP, Taylor BA. FURIN variant associations with postexercise hypotension are intensity and race dependent. Physiological Reports. 2019;7(3):e13952. DOI: 10.14814/phy2.13952

38. Brito LC, Fecchio RY, Peçanha T, AndradeLima A, Halliwill JR, Forjaz CL. Postexercise hypotension as a clinical tool: a "single brick" in the wall. Journal of the American Society of Hypertension. 2018;12(12):e59-e64. DOl: 10.1016/j.jash.2018.10.006

39. Boeno FP, Munhoz SV, Ramis TR, LealMenezes R, Fragoso EB, Müller DC, Teixeira BC, Reischak-Oliveira A. Postexercise hypotension predicts the chronic effects of resistance training in middle-aged hypertensive individuals: a pilot study. Hypertension Research. 2021;44(5):598-600. DOI: 10.1038/s41440-020-00596-9

40. Kobayashi J, Murase Y, Asano A, Nohara A, Kawashiri MA, Inazu A, Yamagishi $M$, Mabuchi $\mathrm{H}$. Effect of walking with a pedometer on serum lipid and adiponectin levels in Japanese middle-aged men. Journal of Atherosclerosis and Thrombosis. 2006;13(4):197-201. DOI: 10.5551/jat.13.197

41. Dow CA, Stauffer BL, Brunjes DL, Greiner JJ, DeSouza CA. Regular aerobic exercise reduces endothelin-1-mediated vasoconstrictor tone in overweight and obese adults. Exp Physiol. 2017;102(9):11331142. DOI: 10.1113/EP086454

42. Yáñez-Sepúlveda R, Barraza-Gómez F, BaezSan Martin E, Araneda OF, Zavala JP, Hecht GK, et al. Differences in energy expenditure, amount of physical activity and physical exertion level during a Zumba fitness class among adult women who are normal weight, overweight and obese. The Journal of Sports Medicine and Physical Fitness. 2018;58(1-2):113-119. DOI: 10.23736/S00224707.17.06835-9

43. Aznar Laín S, Webster T. Actividad física y salud en la infancia y la adolescencia. Guía para todas las personas que participan en su educación. 1ra Edición. Madrid. Grafo. 2009. $109 \mathrm{p}$.

44. Achari AE, Jain SK. Adiponectin, a therapeutic target for obesity, diabetes, and endothelial dysfunction. International Journal of Molecular Sciences. 2017;18(6):1321-37. DOI: 10.3390/ ijms18061321

45. do Vale Moreira NC, Montenegro RM, Meyer HE, Bhowmik B, Mdala I, Siddiquee T, et al. Glycated hemoglobin in the diagnosis of diabetes mellitus in a semi-urban Brazilian population. Int J Environ Res Public Health. 2019;16(19):3598-612. DOI: 10.3390/ ijerph16193598

46. Yom-Tov E, Feraru G, Kozdoba M, Mannor S, Tennenholtz M, Hochberg I. Encouraging physical activity in patients with diabetes: intervention using a reinforcement learning system. J Med Internet Res. 2017;19(10):e338. DOI: 10.2196/jmir.7994

47. Horáková D, Štěpánek L, Janout V, Janoutová J, Pastucha D, Kollárová H, et al. Optimal homeostasis model assessment of insulin resistance (HOMA-IR) cut-offs: A crosssectional study in the Czech population. Medicina (Kaunas). 2019;55(5):158-65. DOI: 10.3390/medicina55050158

48. Dasgupta K, Rosenberg E, Joseph L, Cooke A, Trudeau L, Bacon S, et al. Physician step prescription and monitoring to improve ARTERial health (SMARTER): A randomized controlled trial in patients with type 2 diabetes and hypertension. Diabetes Obes Metab. 2017;19(5):695-704. DOI: 10.1111/ dom.12874

49. Huh U, Tak YJ, Song S, Chung SW, Sung SM, Lee CW, et al. Feedback on physical activity through a wearable device connected to a mobile phone app in patients with metabolic syndrome: Pilot study. JMIR mHealth and uHealth. 2019 Jun 18;7(6):e13381. DOI: 10.2196/13381 